\title{
Non-invasive current stimulation in vision recovery: a review of the literature
}

\author{
Cecilia Perinn $^{\mathrm{a}, \mathrm{b}, *}$, Barbara Viganò ${ }^{\mathrm{c}}$, Daniele Piscitellia ${ }^{\mathrm{a}, \mathrm{d}}$, Barbara Maria Matteo ${ }^{\mathrm{a}}$, \\ Roberto Meroni ${ }^{\mathrm{a}, \mathrm{b}, \mathrm{e}}$ and Cesare Giuseppe Cerri ${ }^{\mathrm{a}, \mathrm{b}}$ \\ ${ }^{a}$ Dipartimento di Medicina e Chirurgia (School of Medicine and Surgery) University of Milan-Bicocca, \\ Milan, Italy \\ ${ }^{\mathrm{b}}$ Milan Center for Neuroscience (NeuroMI), University of Milan-Bicocca, Milan, Italy \\ ${ }^{\mathrm{c}}$ Istituti Clinici Zucchi, Carate Brianza (MB), Italy \\ ${ }^{\mathrm{d}}$ School of Physical and Occupational Therapy, McGill University, Montreal, QC, Canada \\ ${ }^{\mathrm{e}}$ Current Affilation: Department of Physiotherapy, LUNEX International University of Health, \\ Exercise and Sports. Differdange, Luxembourg
}

\begin{abstract}
.
Background: Around 253 million people worldwide suffer from irreversible visual damage. Numerous studies have been carried out in order to unveil the effects of electrical stimulation (ES) as a useful tool for rehabilitation for different visual conditions and pathologies.

Objective: This systematic review aimed to 1) examine the current evidence of ES efficacy for the treatment of visual pathologies and 2) define the corresponding degree of the recommendation of different ES techniques.

Methods: A systematic review was conducted in MEDLINE and Cochrane Library database to collect documents published between 2000 and 2018. For each study, Level of Evidence of Effectiveness of ES as well as the Class of Quality for the treatment of different visual pathologies were determined.

Results: Thirty-eight articles were included. Studies were grouped according to the pathology treated and the type of stimulation administered. The first group included studies treating pre-chiasmatic pathologies (age-related macular degeneration, macular dystrophy, retinal artery occlusion, retinitis pigmentosa, glaucoma, optic nerve damage, and optic neuropathy) using pre-chiasmatic stimulation; the second group included studies treating both pre-chiasmatic pathologies (amblyopia, myopia) and post-chiasmatic pathologies or brain conditions (hemianopsia, brain trauma) by means of post-chiasmatic stimulation. In the first group, repetitive transorbital alternating current stimulation (rtACS) reached level A recommendation, and transcorneal electrical stimulation (tcES) reached level B. In the second group, both high-frequency random noise stimulation (hf-RNS) and transcranial direct current stimulation (tDCS) reached level C recommendation.

Conclusions: Study's findings suggest conclusive evidence for rtACS treatment. For other protocols results are promising but not conclusive since the examined studies assessed different stimulation parameters and endpoints. A comparison of the effects of different combinations of these variables still lacks in the literature. Further studies are needed to optimize existing protocols and determine if different protocols are needed for different diseases.
\end{abstract}

Keywords: Electrical stimulation, transcorneal electrical stimulation (tcES), repetitive transorbital alternating current stimulation (rtACS), high-frequency random noise stimulation (hf-RNS), transcranial direct current stimulation (tDCS), visual damage, hypovision

\footnotetext{
*Corresponding author: Cecilia Perin, Dipartimento di Medicina e Chirurgia (School of Medicine and Surgery), University of Milan-Bicocca, via Cadore 48, 20900 Monza (MB), Italy. Tel.: +390362986446; Fax: +390362986439; E-mail: cecilia.perin@ unimib.it.
}

\section{Introduction}

Since the 18th century, the effects of an electric current flow inside the human body have been studied with interest and curiosity. Presently, the scientific community has been approaching these 
investigations using new methods, with the purpose of broadening the application possibilities of electrical stimulation (ES) (Bindman et al., 1964). In fact, there has been a flourishing development of techniques for transcranial ES, employing various methods and durations of treatment, which have proven to be effective in the treatment of a wide range of pathologies (schizophrenia, stroke, fibromyalgia, etc.) (Lefaucheur et al., 2017).

In particular, it is known that the main effect of ES in the brain is a decrease of the membrane potential that causes hyperpolarization or depolarization in relation to the orientation of the flow of current with respect to the axonal orientation. Neural networks respond to current fields more selectively than single neurons, and the current flow can interfere with functional connectivity, synchronization, and oscillatory activity in various cortical and subcortical networks (Purpura \& McMurtry,1965). In current protocols, the applied intensity of ES is low (less than $4 \mathrm{~mA}$ ) (Bikson et al., 2016). The shape of the wave can be different, depending on the amplitude, frequency and duration of the stimulation. In direct current stimulation, the current intensity and polarity can be constant in time; in alternating current stimulation and in random noise stimulation, polarity and intensity vary in time specifically and randomly, respectively. The current is applied to the body by electrodes: it flows into the body from the anode and out of it from the cathode. The anodal current is considered to have excitatory effects on the brain, while the cathodal current is considered to have inhibitory effects (Purpura $\&$ McMurtry, 1965).

In the 2000s, numerous studies on the effects of weak current flows on the visual system have been published (Antal et al., 2011; Kanai et al., 2008). Several tests have been performed on the healthy visual system, and it has been observed that the stimulation induced by a flow of current may reach the conscious level, producing the appearance of phosphenes. Electroencephalography (EEG) has revealed changes in ocular neurophysiology characterized by increased connectivity. The functional changes observed following ES include changes in visual acuity (VA), visual field (VF), contrast sensitivity (CS), and color discrimination (Fedorov et al., 2011). If ES can influence the healthy visual brain, it is reasonable to ask whether it could affect a damaged visual system and could be useful in restoring the visual function.

The first approach to treat a visual impairment by means of current stimulation was described by Shinoda et al. (2008). This preliminary study, conducted in age-related macular degeneration (AMD) patients, demonstrated an improvement of best-corrected VA after transcorneal alternating current stimulation. Since then, numerous studies have been carried out in order to determine the effects of ES in many visual conditions and pathologies. However, there is no standard protocol to date for the application of this technique to treat different visual pathologies.

The purpose of this review is to describe the state of the art of this treatment, defining its efficacy and therapeutic benefits in various visual pathologies.

\section{Methods}

A comprehensive literature search from 2000 to 2018 was performed in order to identify original articles describing retrospective and prospective studies. The following electronic databases were used: Medline (Pubmed research engine) and the Cochrane Library. Additional records were searched on the reference list of the included articles and by hand searching related studies to retrieved other relevant articles (i.e., cross-referencing). The search was based on three algorithms highlighting the effects of current stimulation produced on vision by the three existing non-invasive brain stimulation (NIBS) techniques: alternating current stimulation (search algorithm: "Alternating current stimulation" AND "vision" OR "ocular"), direct current stimulation (search algorithm: "Direct current stimulation" AND "vision" OR "ocular"), and random noise stimulation (search algorithm: "Random noise stimulation" AND "vision” OR “ocular”).

A preliminary screening of publications was made in order to eliminate duplications, articles not written in English, and articles that were not pertinent to the subject. The articles were examined on the basis of the following eligibility criterion: the articles had to describe primary scientific research (i.e., reviews, meta-analyses, state-of-the-art articles, and letters to the editor were excluded) on the use of NIBS in humans with any kind of visual impairment. Information on methodology and results was extracted. From each study, the number of patients, condition treated, overall outcome, and side effects were obtained.

Two independent reviewers critically read all selected publications and classified them according to criteria derived from those proposed by the European Federation of Neurological Societies (Brainin et al., 2004). Studies were classified by their methodological quality from Class I to 
Class IV, according to Lefaucheur et al. (2017) recommendation. Briefly, Class I: a randomized, placebo-controlled clinical trial with blind outcome assessment in $n \geq 25$ patients receiving active treatment. The study design should include (a) randomization concealment, clearly identified (b) primary outcomes and (c) exclusion/inclusion criteria, (d) acceptable dropouts and crossovers which can lead to minimal biased results and (e) essential baseline characteristics similar between the intervention and control group or using statistical adjustment accounting for differences when appropriate. Class II: randomized, placebo-controlled trial with $10 \leq n<25$ $\mathrm{n}$ patients or without one of the a-e criteria. Class III: controlled trials, with $n<10$, or $n>10$ but showing flaws in the study design. Class IV non- controlled studies, case series, and case reports.

On the basis of the number of studies carried out and their Class of Quality, we determined a Level of Evidence of Effectiveness of the different current stimulation treatments for each visual pathology. Level A of effectiveness was attributed to a therapy that resulted in being definitely effective (literature reporting two Class I studies or one Class I study and at least two Class II studies); Level B was attributed to a therapy appearing as probably effective (literature reporting one Class I study and less than two Class II studies, or at least two Class II studies, or one Class II study and at least two Class III studies); Level C was attributed to a therapy appearing as possibly effective (literature reporting one Class II study and less than two Class III studies, or at least two Class III studies) (Lefaucheur et al., 2017).

\section{Results}

\subsection{Study identification and quality assessment}

The search process identified 1048 documents: 91 were duplicate publications, 354 were excluded because the main topic was not visual defect, 239 were discarded because the participants were animals, 83 were reviews and, therefore, were excluded, 10 studies were excluded because they did not use ES, and 36 papers were not available in English. In addition, 122 studies were excluded because they used subretinal electrical stimulation (SES), which cannot be considered a non-invasive ES technique. The fulltext articles of the 113 remaining trials were obtained. After excluding the studies considering healthy subjects and/or not using ES in the clinical practice as

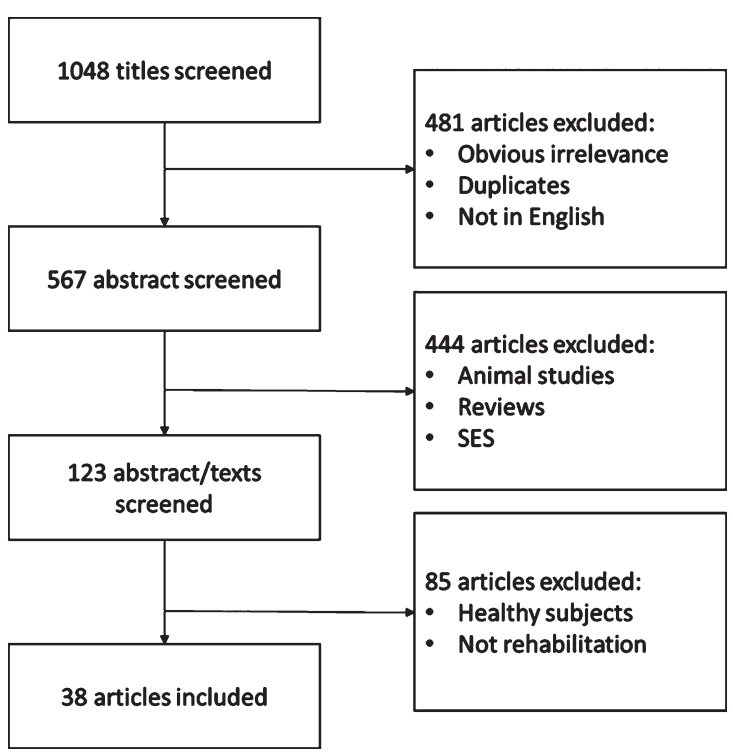

Fig. 1. Flow diagram of study selection. $\mathrm{SES}=$ Subretinal Electrical Stimulation.

a rehabilitation technique, 38 studies were subjected to the final review. The results of the selection process are shown in Fig. 1 (For further details on study charateristcs and Level of Evidence, see Appendix 1).

The studies included in the analysis were grouped on the basis of the anatomical site of current stimulation and the pathology treated (see Table 1).

As shown in the Table 1, the pathologies treated were progressive/degenerative, vascular, or dysfunctional, and different stimulation protocols were used.

With reference to the pre-chiasmatic stimulation site, three application areas were identified: cornea (transcorneal alternating current stimulation, tcES), eyelid (transpalpebral alternating current stimulation, tpES), and periorbital zone (rtACS).

In tcES, the electrodes are positioned on the ocular surface when the patient is wearing a lens frame (Fujikado et al., 2006). This alternating microcurrent stimulation is suggested to act via several mechanisms: neurotrophic, anti-apoptotic, anti-glutammatic, and anti-inflammatory (Sehic et al., 2016). For this reason, tcES is used to treat nonarteritic ischemic optic neuropathy (NAION) (Fujikado et al., 2006), retinal artery occlusion (RAO) (Inomata et al., 2007; Oono et al., 2011; Naycheva et al., 2013), traumatic optic neuropathy (TON) (Fujikado et al., 2006), retinitis pigmentosa (Schatz et al., 2011, 2017; Robles-Camarillo et al., 2013; Bittner et al., 2018a, 2018b), and macular dystrophy (Ozeki et al., 2013). We examined 11 papers on tcES. Five of these 
Table 1

Subdivision of the studies in relation to the site of stimulation and the treated pathology

\begin{tabular}{|c|c|c|c|}
\hline Site of stimulation & $\begin{array}{c}\text { Kind of } \\
\text { stimulation }\end{array}$ & Clinical Conditions & $\begin{array}{l}\text { First Author and } \\
\text { year }\end{array}$ \\
\hline Pre-chiasmatic & $\begin{array}{c}\text { rtACS } \\
\text { tpES } \\
\text { tcES }\end{array}$ & $\begin{array}{l}\text { Age-related macular } \\
\quad \text { degeneration } \\
\text { Macular dystrophy } \\
\text { Retinal artery occlusion } \\
\text { Retinitis Pigmentosa } \\
\text { Glaucoma } \\
\text { Optic nerve damage } \\
\text { Optic neuropathy }\end{array}$ & $\begin{array}{l}\text { Gall et al,2010; Fedorov et al., 2011; Gall et al, 2011; Sabel } \\
\text { et al., 2011; Schmidt et al., 2013; Bola et al., 2014; Gall et } \\
\text { al., 2016; Shinoda et al., 2008; Anastassiou et al., 2013; } \\
\text { Chaikin et al., 2015; Gil-Carrasco et al., 2018; Fujikado } \\
\text { et al., 2006; Inomata et al., 2007; Oono et al., 2011; } \\
\text { Schatz et al., 2011; Naycheva et al., 2013; Ozeki et al., } \\
\text { 2013; Robles-Camarillo et al., 2013; Schatz et al., 2017; } \\
\text { Bittner et al., 2018; Bittner et al., 2018; Ota et al., } 2018\end{array}$ \\
\hline Post-chiasmatic & $\begin{array}{c}\text { hf-tRNS } \\
\text { tDCS }\end{array}$ & $\begin{array}{l}\text { Visual field loss } \\
\text { Post-stroke visual perception } \\
\text { degradation } \\
\text { Amblyopia } \\
\text { Myopia }\end{array}$ & $\begin{array}{l}\text { Camilleri et al, 2014; Campana et al., 2014; Camilleri et al., } \\
\text { 2016; Moret et al., 2018; Halko et al., 2011; Plow et al., } \\
\text { 2011; Plow et al., 2012a; Plow et al., 2012b; Cowey et al., } \\
\text { 2013; Olma et al., 2013; Spiegel et al., 2013; Spiegel et } \\
\text { al., 2013; Ding et al., 2016; Kim et al., 2016; Alber et al., } \\
\text { 2017; Matteo et al., 2017 }\end{array}$ \\
\hline
\end{tabular}

Notes: rtACS: repetitive transorbital alternating current stimulation, tpES: transpalpebral electrical stimulation, tcES: transcorneal electrical stimulation, hf-tRNS: high-frequency random noise stimulation, tDCS: transcranial direct current stimulation.

studies used this technique to treat retinitis pigmentosa. Schatz et al. $(2011,2017)$ studies, classified as Class II and III, chose VF area improvement as the primary endpoint and showed a trend of prevention of VF loss in patients stimulated with 150 and $200 \%$ of their individual electrical phosphene threshold and an improvement in electroretinogram waves. Bittner et al. (2018a, 2018b), in their Class III and IV studies, showed a significant improvement in retrobulbar central retinal artery flow, an increment in the retinal blood flow, and visual function improvements in some patients, which lasted for several months. In their Class IV paper, Robles-Camarillo et al. (2013) obtained the same tendency of VF improvement. For these reasons, a level B recommendation is proposed for tcES use in the treatment of retinitis pigmentosa. For NAION, TON, and macular dystrophy, the available data were not sufficient to express a level of recommendation.

The transpalpebral electronic stimulator is based on a digital adaptive model that delivers electronic waveforms specific for transpalpebral electrical stimulation (tpES). TpES is shown to act via the same mechanisms as tcES, with an additional effect on intraocular pressure via $\mathrm{BKCa}^{2}$ channel modulation. For this reason, it has also been suggested for glaucoma treatment (Gil-Carrasco et al., 2018). In three articles, tpES was used to treat AMD. In a Class II study by Anastassiou et al. (2013), a temporary increase in visual function in some patients was achieved. Other authors obtained Class IV results; therefore, no level of recommendation can be assigned for tpES use.
The technique rtACS consists of a multi-channel device delivering weak periorbital oscillatory current sinus-pulses over four individual periorbital electrodes. In general, the four superficial stimulating electrodes are attached bilaterally, above, and below to the eye. The proposed mechanism of action is the neuromodulation of oscillatory brain activity, as demonstrated by the observed changes in alfa-band brain activity, leading to synchronization. According to the "residual vision activation theory," the synchronization of central nervous pathways (within-systems and network plasticity) may improve the injured VF (Sabel et al., 2011b). For these reasons, rtACS has been studied to treat optic nerve damage. We found one particular Class I study carried out by Gall et al. (2016) involving people with different pathologies (91 subjects with glaucoma, anterior ischemic optic neuropathy, and other types of optic atrophy). The results showed that rtACS induced a mean improvement of the VF of $24.0 \%$, significantly higher than that observed after sham stimulation $(2.5 \%)$. This improvement persisted for at least two months. Moreover, Sabel et al. (2011a) showed, in a Class II study, that rtACS vision restoration was possible also many years after optic neuropathy. Furthermore, Gall et al. (2011), in their Class II study, found that rtACS enhanced vision restoration after unilateral, longterm optic nerve lesions. Schmidt et al. (2013), in their Class III paper, tried to test if the improvement of the perimetry assessed as detection accuracy in defective visual field sectors using a computer-based highresolution perimetry, was associated with changes in alpha-synchronization but failed to demonstrate it. 
These data are consistent with those of other Class III and Class IV studies.

Post-chiasmatic stimulation involves the use of two techniques: high-frequency transcranial random noise stimulation (hf-tRNS) on the occipital lobe and transcranial direct current stimulation (tDCS) on the middle temporal, occipital, or C3 (M1) areas.

The hf-tRNS technique uses an alternating current with random frequencies delivered during task execution. It seems to act by increasing the activity of ion (sodium) channels and, therefore, by promoting a temporal summation of small membrane potentials induced by consecutive openings of these channels. The technique utilizes a battery-driven stimulator acting through a pair of saline-soaked sponge electrodes placed on the scalp over the area of interest (active electrode) and a reference electrode. The hf-tRNS has been proposed for the treatment of amblyopia (Campana et al., 2014; Moret et al., 2018) and of refractive defects (e.g., myopia, Camilleri et al., 2016). To our knowledge, three Class III and one Class IV papers were published, allowing us to assign a level $\mathrm{C}$ of recommendation for hf-tRNS use in conditions such as amblyopia and myopia, with associated training. In particular, Camilleri et al. (2014) studied the effect of hf-RNS on myopia, showing that hf-RNS associated with training (contrast detection task) improved CS and VA when compared to training alone. Camilleri et al. (2016) then tested 30 myopic patients, assigning them to three groups: the first underwent training associated with hf-RNS, the second underwent training associated with sham hfRNS, and the third underwent hf-RNS alone. The results showed that training improved VA and CS when coupled with hf-RNS, while marginal effects on CS were obtained with the sole administration of hf-RNS.

The tDCS technique directly modulates the transmembrane potential (thus, the firing rate) of individual neurons with a continuous flux of current that produces initial facilitation, often followed by an adaptation to rebalance the modulation of ion channel conductance. This technique is the most used in neurological rehabilitation to boost brain recovery after stroke, head trauma, or inflammatory conditions such as multiple sclerosis (Lefaucheur et al., 2017); therefore, it is not surprising that it has been proposed for conditions such as amblyopia or hemianopia. In amblyopia patients, tDCS demonstrated effectiveness in improving contrast, stereopsis, motion perception, VF, and visual evoked potential (VEP). In a Class III study, Ding et al. (2016) found a specific effect of anodal tDCS in increasing VEP amplitude and current stimulation in amblyopic eyes. They concluded that anodal tDCS alone might have clinical relevance for the treatment of amblyopia in adulthood. Spiegel et al. (2013a, 2013b) compared the effects of dichoptic treatment alone and dichoptic treatment combined with visual cortex tDCS on binocular (stereopsis) and monocular (visual acuity) visual function. The combined treatment led to more significant improvements in stereoacuity than the dichoptic treatment alone, indicating that direct current stimulation of the visual cortex boosts the efficacy of dichoptic video game-based treatment. These results allow assigning a level $\mathrm{C}$ recommendation for the treatment of amblyopia by tDCS. In several studies, tDCS was shown to be effective also in hemianopia. We collected five Class III studies, corresponding to level $\mathrm{C}$ of recommendation. Three studies associated tDCS with training (vision restoration therapy) in hemianopic patients (Plow et al., 2012a, 2012b; Alber et al., 2017) and compared two groups of patients: one group treated with training alone, the other group treated with training associated with tDCS. Plow et al. studies (2012a, 2012b) recruited four and six patients per group, respectively, whereas Alber et al. (2017) study enrolled seven patients per group. Plow et al. (2012a, 2012b) showed an accelerated recovery in the VF and a greater shift of the VF border when tDCS was associated with training. Alber et al. (2017) showed a higher recovery in the percentage of change of VF stimulus detection when tDCS was combined with vision restoration training. Both results showed a facilitation role of tDCS in the treatment of hemianopia when associated with training. Olma et al. (2013) applied anodal tDCS over the visual cortex and observed an improvement in motion perception, still measurable after 14 and 28 days. Kim et al. (2016) found no significant difference between tDCS stimulation plus occupational therapy (OT) and OT alone in post-stroke patients suffering from visual perception deficit.

\subsection{Stimulation parameters}

In Table 2, stimulation parameters for all types of stimulation used in the examined studies are reported.

Two main variables should be considered in the context of ES: the position of the electrodes and the stimulation parameters.

For rtACS and tpES, 11 studies placed the stimulating electrodes (one or more) close to the orbit of 
Table 2

Stimulation parameters used in the analyzed studies

\begin{tabular}{|c|c|c|c|c|c|c|c|c|}
\hline $\begin{array}{l}\text { Type of } \\
\text { stimulation }\end{array}$ & $\begin{array}{l}\text { Side of } \\
\text { stimulation }\end{array}$ & $\begin{array}{l}\text { Number } \\
\text { of } \\
\text { studies }\end{array}$ & $\begin{array}{l}\text { Number } \\
\text { of } \\
\text { electrodes }\end{array}$ & $\begin{array}{l}\text { Position of } \\
\text { electrodes }\end{array}$ & $\begin{array}{l}\text { Quantity of } \\
\text { current }\end{array}$ & $\begin{array}{l}\text { Time } \\
\text { of } \\
\text { stimulation }\end{array}$ & $\begin{array}{l}\text { Number } \\
\text { of } \\
\text { sessions }\end{array}$ & $\begin{array}{l}\text { On-/ } \\
\text { off- } \\
\text { set }\end{array}$ \\
\hline rtACS & $\begin{array}{r}\text { Pre-chiasmatic } \\
\text { (transorbital) }\end{array}$ & 7 & 4 & $\begin{array}{l}\text { Transorbital (both } \\
\text { eyes) }\end{array}$ & $\begin{array}{l}\text { From } 115 \text { to } \\
1000 \mu \mathrm{A} ; \\
\text { from } 5 \text { to } \\
37 \mathrm{~Hz}\end{array}$ & $\begin{array}{l}\text { From } 20 \text { to } \\
50 \mathrm{~min}\end{array}$ & 10 & 7 off-set \\
\hline tpES & $\begin{array}{l}\text { Pre-chiasmatic } \\
\text { (transpalpe- } \\
\text { bral) }\end{array}$ & 4 & 2 or 8 & $\begin{array}{l}\text { Transpalpebral } \\
\text { (both eyes) }\end{array}$ & $\begin{array}{l}\text { From } 100 \mu \mathrm{A} \\
\text { to } 800 \mu \mathrm{A} ; \\
\text { from } 0.28 \text { to } \\
290 \mathrm{~Hz}\end{array}$ & $\begin{array}{l}\text { From } 320 \mathrm{sec} \\
\text { to } 40 \mathrm{~min}\end{array}$ & From 2 to 30 & 4 off-set \\
\hline tcES & $\begin{array}{l}\text { Pre-chiasmatic } \\
\quad \text { (transcorneal) }\end{array}$ & 11 & 1 or 2 & $\begin{array}{l}\text { Cornea (one or } \\
\text { both eye) }\end{array}$ & $\begin{array}{l}\text { From } 100 \mu \mathrm{A} \\
\text { to } 1.75 \mathrm{~mA} \text {; } \\
\text { from } 11.8 \text { to } \\
20 \mathrm{~Hz}\end{array}$ & $\begin{array}{l}\text { From } 30 \text { to } \\
\quad 45 \mathrm{~min}\end{array}$ & From 1 to 27 & 11 off-set \\
\hline hf-tRNS & $\begin{array}{l}\text { Post-chiasmatic } \\
\text { (transcranial) }\end{array}$ & 4 & 2 & $\begin{array}{l}\mathrm{Oz}+\text { upper right } \\
\text { arm; } \\
\mathrm{Oz}+\text { forehead }\end{array}$ & $\begin{array}{l}1,5 \mathrm{~mA}(0 \mathrm{~mA} \\
\text { offset }) \\
100-640 \mathrm{~Hz}\end{array}$ & $25 \mathrm{~min}$ & 8 & 4 on-set \\
\hline tDCS & $\begin{array}{l}\text { Post-chiasmatic } \\
\text { (transcranial) }\end{array}$ & 12 & 2 & $\begin{array}{l}\text { Oz-Cz; left } \\
\text { MT/V5-right } \\
\text { MT/V5 and left } \\
\text { MT/V5-right } \\
\text { frontal pole; } \\
\text { C3-SoA; } \\
\text { O1/O2-Cz; } \\
\text { PO3/PO4-SoA }\end{array}$ & $\begin{array}{l}\text { From } 1 \text { to } \\
2 \mathrm{~mA}\end{array}$ & $\begin{array}{l}\text { From } 15 \text { to } \\
30 \mathrm{~min}\end{array}$ & From 2 to 72 & $\begin{array}{l}11 \text { on-set, } \\
1 \text { off-set }\end{array}$ \\
\hline
\end{tabular}

Notes: SoA = Supraorbital Area.

the eye. Nine out of 11 studies used a montage of four electrodes near the eyeballs and the return electrodes on the right arm (opposite to the heart, in order to avoid a current flow near the heart). Anastassiou et al. (2013) used eight contact points around the eye: four over the upper eyelid and four over the lower eyelid. Shinoda et al. (2008) proposed a montage in which two electrodes (one for each eye) were applied as patches on the eyelids.

The current intensity and frequency varied among the studies. The current frequency is usually related to the phosphene threshold, i.e., the minimal ES that evokes phosphene perceptions in the visual field (Naycheva et al., 2012) aiming at achieving a stimulation intensity about $125 \%$ of the phosphene threshold, and the current intensity is always under $1000 \mu \mathrm{A}$. The current alternates at a relatively low frequency, from 5 to $30 \mathrm{~Hz}$. Several studies suggest a correlation between current frequency and EEG frequency bands (e.g., Bola et al., 2014; Kanai et al., 2008).

In the case of tcES, the electrode is placed directly on the cornea. Two main kinds of the electrode were used for transcorneal stimulation: five studies used a "Dawson, Trick, and Litzkow electrode" (a metalcoated nylon thread that is installed between the medial and the lateral eyelid), and six studies used electrodes embedded into a "contact lens." The stimulation intensity varied among the studies and was usually related to the phosphene threshold. Some authors (Fujikado et al., 2008; Oono et al., 2011; Ozeki et al., 2013; Bittner et al., 2018a) reported an intensity lower than $1100 \mu \mathrm{A}$. The frequency was settled to $20 \mathrm{~Hz}$ in 9 studies out of 11 . No study used a frequency intensity above $100 \mathrm{~Hz}$.

For tDCS, in 7 out of 12 studies, the active electrode was placed over $\mathrm{Oz}$ (according to the 10-20 EEG international reference system), and the reference electrode was placed over Cz. Alber et al. (2017) placed the electrodes in the occipital areas $(\mathrm{O} 1$ or O2), depending on the brain lesion. Cowey et al. (2013) stimulated the MT/V5, positioning the reference electrode in the MT/V5 contralateral area or in the right frontal pole. Matteo et al. (2017) stimulated the parieto-occipital area. tDCS was always delivered at $2 \mathrm{~mA}$ intensity, except for two studies (Cowey et al., 2013; Olma et al., 2013) that delivered it at $1.5 \mathrm{~mA}$.

In the studies that used hf-RNS, the active electrode was placed over $\mathrm{Oz}$, similar to tDCS, but the reference electrode was usually placed on the right arm. The intensity was set above $1 \mathrm{~A} / \mathrm{msq}$, and the 
frequency ranged from 100 to $640 \mathrm{~Hz}$, higher than the parameters used for tDCS.

\subsection{Safety}

When analyzing the studies aimed at restoring the visual system by means of ES, we did not find any serious adverse event (SAE) in a total of 1132 patients. The only adverse events found in the literature related to tDCS for visual impairment were "occasional itching or tingling" (Alber et al., 2017) and "slight tingling sensation under the electrodes" (Spiegel et al., 2013b). It should be pointed out that in all these studies, only one patient withdrew from a trial because of discomfort (Spiegel et al., 2013a). In that study, the authors stated: "there were no adverse effects during or following tDCS." The same level of safety was found analyzing the studies aimed at restoring vision by means of alternating current stimulation (Sabel et al., 2011; Gall et al., 2016; Gall et al., 2011; Fedorov et al., 2011; Schmidt et al., 2013; Gall et al., 2010; Gall et al., 2013; Bola et al., 2014; Anastassiou et al., 2013; Shinoda et al., 2008). Patients treated with rtACS reported cutaneous sensations in rare cases $(<10 \%)$, and they experienced mild headache, general fatigue, dizziness, or blood pressure fluctuations in sporadic cases $(<5 \%)$. The only adverse event considered as "serious" after stimulation with tpES was dermatitis on both superior lids (Shinoda et al., 2008). In that specific case, researchers decided to stop the treatment, despite the patient wishing to continue. The following local side effects were reported for tcES: foreign body sensation, dry eyes (reported in about $3 \%$ and $15 \%$ of cases, respectively), transient superficial keratitis (reported in about $5 \%$ of cases). Side effects were local and transient, with no obvious changes detected by the slit-lamp examination and healed by the next day (Inomata et al., 2007; Ozeki et al., 2013). The hf-RNS technique tested over 73 visually impaired patients (Camilleri et al., 2014; Camilleri et al., 2016; Campana et al., 2014; Moret et al., 2018). None of them reported any side effects during or after treatment. Finally, there was no report of any single SAE in the visual system in the studies using tDCS, tACS, tcES, tpES, and hf-RNS.

Considering the low frequency and the low level of concern of the adverse events reported in more than 1000 visually impaired patients that underwent current stimulation in different institutions according to different treatment protocols, it is possible to state that the use of ES for the treatment of vision loss can be considered safe.

\section{Conclusions}

This review has highlighted that ES is a promising, safe, and effective technique. This work presents for the first time a comprehensive evidence-based analysis of the reported clinical efficacy of various ES montages that could lead to therapeutic applications in the visual domain. According to our analysis, the available data allow making a Level A recommendation (conclusive evidence) for rtACS and a Level C recommendation (possible efficacy) for hfRNS and tDCS for the treatment of several clinical conditions with both ophthalmologic and neurologic impairment.

In the ophthalmological field, it seems that the stimulation with alternating current favors the recovery of patients suffering from untreatable pathologies related to retinal and optic nerve impairment (glaucoma, age-related macular degeneration, retinitis pigmentosa, etc.). On the other hand, within the field of brain impairment (e.g., trauma, stroke, brain tumor), there is evidence of possible beneficial effects of direct current stimulation, whereas no conclusive evidence can be made about the use of alternating current (for a review, see Matteo et al., 2016). Notably, a recent Cochrane review found limited evidence for using tDCS in addition to visual restitution therapy in post-stroke individuals (Pollock et al., 2019). However, more studies are needed to bridge neurophysiology and post-stroke rehabilitation (Piscitelli, 2019).

Many authors have tried to understand how ES can affect the visual system and at what brain level. Sabel et al. (2011) proposed the "residual vision activation theory", according to which non-invasive current applications (transpalpebral, transcorneal, transorbital) provoke visual percepts (phosphenes) in the visual cortex, which may lead to excitability changes in the visual cortex and other brain structures and thus improve the visual functions (Sabel et al., 2011). The theory is based on the fact that the loss of vision in patients suffering from stroke, neurotrauma, glaucoma, amblyopia, and AMD is usually not complete: some structures are typically spared by the damage. By engaging the residual functional structures in repetitive ES, the neural tissue can be reactivated and restored (Sabel et al., 2013). The stimulation may have the effects of strengthening the synaptic 
transmission, synchronizing partially damaged structures (within-systems plasticity), and reconnecting downstream the neuronal networks (network plasticity). It has also been shown that ES modulates the activity of the visual brain: this was observed and recorded by VEP, EEG, and electroretinography. In general, an enhancement of the EEG-alpha activity (i.e., $8-12 \mathrm{~Hz}$ frequency of the EEG spectrum; Berger, 1931) and/or stronger connectivity of the different parts of the visual cortex were registered (Naycheva et al., 2013). Recently, in line with these neurophysiological findings, Sabel et al. (2019) showed in a feasibility study, that transorbital rtACS modified MRI blood oxygenation level-dependent (BOLD) activity upon visual stimulation. Similarly, the same hypothesis can be proposed for postchiasmatic reorganization (for a detailed review, see Matteo et al., 2016). However, ES techniques still present many limits, and some questions remain to be answered. Further research is needed to investigate the effect of training on brain-related low vision (amblyopia and hemianopia). Indeed studies considering the effect of current stimulation in patients with visual brain defects utilized several kinds of rehabilitation training. Moreover, further studies are required to assess different electrodes position, current types, and treatment conditions.

\subsection{Concluding comments}

In conclusion, ES for vision recovery needs futher investigations. At the current state of art, the stimulation parameters and endpoints are too heterogeneous to allow the design of optimal protocols for each pathology. Therefore, if, on one hand, we can state that current stimulation is useful, we would also recommend more research in order to improve the techniques and optimize health outcomes.

\section{Acknowledgments}

The authors want to thank Dr. Gianluca Sorrento for the linguistic revision.

\section{Disclosure of interest}

The authors report no conflicts of interest

\section{References}

Alber, R., Moser, H., Gall, C., \& Sabel, B. A. (2017). Combined transcranial direct current stimulation and vision restoration training in subacute stroke rehabilitation: A pilot study. $P M \&$ R: The Journal of Injury, Function, and Rehabilitation, 9(8), 787-794.

Anastassiou, G., Schneegans, A. L., Selbach, M., \& Kremmer, S. (2013). Transpalpebral electrotherapy for dry age-related macular degeneration (AMD): An exploratory trial. Restorative Neurology and Neuroscience, 31(5), 571-578.

Antal, A., Paulus, W., \& Nitsche, M. A. (2011). Electrical stimulation and visual network plasticity. Restorative Neurology and Nneuroscience, 29(6), 365-374.

Berger, H. (1931). Über das Elektrenkephalogramm des Menschen. Dritte Mitteilung. Archiv für Psychiatrie und Nervenkrankheiten, 94(1) 16-60.

Bikson, M., Grossman, P., Thomas, C., Zannou, A. L., Jiang, J., Adnan, T.,... \& Brunoni, A. R. (2016). Safety of transcranial direct current stimulation: Evidence based update (2016). Brain Stimulation, 9(5), 641-661.

Bindman, L., Lippold, O., Redferan, J. W. (1964). The action of brief polarizing currents on the cerebral cortex of the rat during current flow and in the producting of long-lasting after effect. Journal of Physiology, 172(3), 369-382.

Bittner, A. K., Seger, K., Salveson, R., Kayser, S., Morrison, N., Vargas, P.,... \& Ramella-Roman, J. (2018a). Randomized controlled trial of electro-stimulation therapies to modulate retinal blood flow and visual function in retinitis pigmentosa. Acta Ophthalmologica, 96(3), e366-e376.

Bittner, A. K., \& Seger, K. (2018b). Longevity of visual improvements following transcorneal electrical stimulation and efficacy of retreatment in three individuals with retinitis pigmentosa. Graefe's Archive for Clinical and Experimental Ophthalmology, 256(2), 299-306.

Bola, M., Gall, C., Moewes, C., Fedorov, A., Hinrichs, H., \& Sabel, B. A. (2014). Brain functional connectivity network breakdown and restoration in blindness. Neurology, 83(6), 542-551.

Brainin, M., Barnes, M., Baron, J. C., Gilhus, N. E., Hughes, R., Selmaj, K., \& Waldemar, G. (2004). Guidance for the preparation of neurological management guidelines by EFNS scientific task forces-revised recommendations 2004. European Journal of Neurology, 11(9), 577-581.

Camilleri, R., Pavan, A., \& Campana, G. (2016). The application of online transcranial random noise stimulation and perceptual learning in the improvement of visualfunctions in mild myopia. Neuropsychologia, 89, 225-231.

Camilleri, R., Pavan, A., Ghin, F., Battaglini, L., \& Campana, G. (2014a). Improvement of uncorrected visual acuity and contrast sensitivity with perceptual learning and transcranial random noise stimulation in individuals with mild myopia. Frontiers in Psychology, 5, 1234.

Campana, G., Camilleri, R., Pavan, A., Veronese, A., \& Lo Giudice, G. (2014b). Improving visual functions in adult amblyopia with combined perceptual training and transcranial random noise stimulation (tRNS): A pilot study. Frontiers in Psychology, 5, 1402.

Chaikin, L., Kashiwa, K., Bennet, M., Papastergiou, G., \& Gregory, W. (2015). Microcurrent stimulation in the treatment of 
dry and wet macular degeneration. Clinical ophthalmology (Auckland, N.Z.), 9, 2345-2353.

Cowey, A., Alexander, I., \& Ellison, A. (2013). Modulation of cortical excitability can speed up blindsight but not improve it. Experimental brain research, 224(3), 469-475.

Ding, Z., Li, J., Spiegel, D. P., Chen, Z., Chan, L., Luo, G., Yuan, J.,... \& Thompson B. (2016). The effect of transcranial direct current stimulation on contrast sensitivity and visual evoked potential amplitude in adults with amblyopia. Scientific Reports, 6, 19280.

Fedorov, A., Jobke, S., Bersnev, V., Chibisova, A., Chibisova, Y., Gall, C., \& Sabel, B. A. (2011). Restoration of vision after optic nerve lesions with noninvasive transorbital alternating current stimulation: A clinical observational study. Brain Stimulation, 4(4), 189-201.

Fujikado, T., Morimoto, T., Matsushita, K., Shimojo, H., Okawa, Y., \& Tano, Y. (2006). Effect of transcorneal electrical stimulation in patients with nonarteritic ischemic optic neuropathy or traumatic optic neuropathy. Japanese Journal of Ophthalmology, 50(3), 266-273.

Gall, C., Fedorov, A. B., Ernst, L., Borrmann, A., \& Sabel, B. A. (2010). Repetitive transorbital alternating current stimulation in optic neuropathy. NeuroRehabilitation, 27(4), 335-341.

Gall, C., Sgorzaly, S., Schmidt, S., Brandt, S., Fedorov, A., \& Sabel, B. A. (2011). Noninvasive transorbital alternating current stimulation improves subjective visual functioning and vision-related quality of life in optic neuropathy. Brain Stimulation, 4(4), 175-188.

Gall, C., Schmidt, S., Schittkowski, M. P., Antal, A., Ambrus, G. G., Paulus, W.,... \& Sabel, B. A. (2016). Alternating current stimulation for vision restoration after optic nerve damage: A randomized clinical trial. PloS one, 11(6), e0156134.

Gall, C., Silvennoinen, K., Granata, G., de Rossi, F., Vecchio, F., Brösel, D.,... \& Sabel, B. A. (2015). Non-invasive electric current stimulation for restoration of vision after unilateral occipital stroke. Contemporary Clinical Trials, 43, 231-236.

Gil-Carrasco, F., Ochoa-Contreras, D., Torres, M. A., SantiagoAmaya, J., Pérez-Tovar, F. W., Gonzalez-Salinas, R., \& Nino-de-Rivera, L. (2018). Transpalpebral Electrical Stimulation as a Novel Therapeutic Approach to Decrease Intraocular Pressure for Open-Angle Glaucoma: A Pilot Study. Journal of Ophthalmology, 2018, 2930519.

Halko, M. A., Datta, A., Plow, E. B., Scaturro, J., Bikson, M., \& Merabet, L. B. (2011). Neuroplastic changes following rehabilitative training correlate with regional electric field induced with tDCS. Neuroimage, 57(3), 885-891.

Inomata, K., Shinoda, K., Ohde, H., Tsunoda, K., Hanazono, G., Kimura, I., ...\& Miyake, Y. (2007). Transcorneal electrical stimulation of retina to treat longstanding retinal artery occlusion. Graefe's Archive for Clinical and Experimental Ophthalmology, 245(12), 1773-1780.

Kanai, R., Chaieb, L., Antal, A., Walsh, V., \& Paulus, W. (2008). Frequency-Dependent Electrical Stimulation of the Visual Cortex. Current Biology, 18(23), 1839-1843.

Kim, K. U., Kim, S. H., \& An, T. G. (2016). Effect of transcranial direct current stimulation on visual perception function and performance capability of activities of daily living in stroke patients. Journal of Physical Therapy Science, 28(9), 25722575.
Lefaucheur, J. P., Antal, A., Ayache, S. S., Benninger, D. H., Brunelin, J., Cogiamanian, F., \& ...Paulus, W. (2017). Evidence-based guidelines on the therapeutic use of transcranial direct current stimulation (tDCS). Clinical Neurophysiology, 128(1), 56-92.

Matteo, B. M., Viganò, B., Cerri, C. G., \& Perin, C. (2016). Visual field restorative rehabilitation after brain injury. Journal of Vision, 16(11), 1-18.

Matteo, B. M., Viganò, B., Cerri, C. G., Meroni, R., Cornaggia, C. M., \& Perin, C. (2017). Transcranial direct current stimulation (tDCS) combined with blindsight rehabilitation for the treatment of homonymous hemianopia: A report of two-cases. Journal of Physical Therapy Science, 29(9), 1700-1705.

Moret, B., Camilleri, R., Pavan, A., Lo Giudice, G., Veronese, A., Rizzo, R., \& Campana G. (2018). Differential effects of highfrequency transcranial random noise stimulation (hf-tRNS) on contrast sensitivity and visual acuity when combined with a short perceptual training in adults with amblyopia. $\mathrm{Neu}$ ropsychologia, 114, 125-133.

Morimoto, T., Fukui, T., Matsushita, K., Okawa, Y., Shimojyo, H., Kusaka, S., ...\& Fujikado, T. (2006). Evaluation of residual retinal function by pupillary constrictions and phosphenes using transcorneal electrical stimulation in patients with retinal degeneration. Graefe's Archive for Clinical and Experimental Ophthalmology, 244(10), 1283-1292.

Naycheva, L., Schatz, A., Röck, T., Willmann, G., Messias, A., Bartz-Schmidt, K. U., ...\& Gekeler, F. (2012). Phosphene thresholds elicited by transcorneal electrical stimulation in healthy subjects and patients with retinal diseases. Investigative Ophthalmology \& Visual Science, 53(12), 7440-7448.

Naycheva, L., Schatz, A., Willmann, G., Bartz-Schmidt, K. U., Zrenner, E., Röck, T., \& Gekeler, F. (2013). Transcorneal Electrical Stimulation in Patients with Retinal Artery Occlusion: A Prospective, Randomized, Sham-Controlled Pilot Study. Ophthalmology and Therapy, 2(1), 25-39.

Olma, M. C., Dargie, R. A., Behrens, J. R., Kraft, A., Irlbacher, K., Fahle, M., \& Brandt, S. A. (2013). Long-term effects of serial anodal tDCS on motion perception in subjects with occipital stroke measured in the unaffected visual hemifield. Frontiers in Human Neuroscience, 24(7), 314.

Oono, S., Kurimoto, T., Kashimoto, R., Tagami, Y., Okamoto, N., \& Mimura, O. (2011). Transcorneal electrical stimulation improves visual function in eyes with branch retinal artery occlusion. Clinical ophthalmology (Auckland, N.Z.), 5, 397-402.

Ota, Y., Ozeki, N., Yuki, K., Shiba, D., Kimura, I., Tsunoda, K., ...\& Tsubota, K. (2018). The Efficacy of Transcorneal Electrical Stimulation for the Treatment of Primary Open-angle Glaucoma: A Pilot Study. The Keio Journal of Medicine, 67(3), 45-53.

Ozeki, N., Shinoda, K., Ohde, H., Ishida, S., \& Tsubota, K. (2013). Improvement of visual acuity after transcorneal electrical stimulation in case of Best vitelliform macular dystrophy. Graefe's Archive for Clinical and Experimental Ophthalmology, 251(7), 1867-1870.

Piscitelli, D. (2019). Neurorehabilitation: Bridging neurophysiology and clinical practice. Neurological Sciences, 40(10), 2209-2211.

Pollock, A., Hazelton, C., Rowe, F. J., Jonuscheit, S., Kernohan, A., Angilley, J., ...\& Campbell, P. (2019). Interventions for 
visual field defects in people with stroke. The Cochrane Database of Systematic Reviews, 5(5), CD008388.

Plow, E. B., Obretenova, S. N., Fregni, F., Pascual-Leone, A., \& Merabet, L. B. (2012a). Comparison of visual field training for hemianopia with active versus sham transcranial direct cortical stimulation. Neurorehabilitation and Neural Repair, 26(6), 616-626.

Plow, E. B., Obretenova, S. N., Halko, M. A., Kenkel, S., Jackson, M. L., Pascual-Leone, A., \& Merabet, L. B. (2011). Combining visual rehabilitative training and noninvasive brain stimulation to enhance visual function in patients with hemianopia: A comparative case study. $P M \& R$ : The Journal of Injury, Function, and Rehabilitation, 3(9), 825-835.

Plow, E. B., Obretenova, S. N., Jackson, M. L., \& Merabet, L. B. (2012b). Temporal profile of functional visual rehabilitative outcomes modulated by transcranial direct current stimulation. Neuromodulation, 15(4), 367-373.

Purpura, D. P., \& McMurtry, J. G. (1965). Intracellular activities and evoked potential changes during polarization of motor cortex. Journal of Neurophysiology, 28, 166-185.

Robles-Camarillo, D., Niño-de-Rivera, L., López-Miranda, J., GilCarrasco, F., \& Quiroz-Mercado, H. (2013). The effect of transcorneal electrical stimulation in visual acuity: Retinitis pigmentosa. Journal of Biomedical Science and Engineering, 6(10), 1-7.

Sabel, B. A., Fedorov, A. B., Naue, N., Borrmann, A., Herrmann, C., \& Gall C. (2011). Non-invasive alternating current stimulation improves vision in optic neuropathy. Restorative Neurology and Neuroscience, 29(6), 493-505.

Sabel, B. A., Henrich-Noack, P., Fedorov, A., \& Gall, C. (2011). Vision restoration after brain and retina damage: The residual vision activation theory. Progress in Brain Research, 192, 199-262.

Sabel, B. A., Kruse, R., Wolf, F., \& Guenther, T. (2013). Local topographic influences on vision restoration hot spots after brain damage. Restorative Neurology and Neuroscience, 31(6), 787-803.

Sabel, B. A., Hamid, A. I. A., Borrmann, C., Speck, O., \& Antal, A. (2019). Transorbital alternating current stimulation modifies BOLD activity in healthy subjects and in a stroke patient with hemianopia: A 7 Tesla fMRI feasibility study. International Journal of Psychophysiology, S0167-8760(18) 31055-31059.

Schatz, A., Röck, T., Naycheva, L., Willmann, G., Wilhelm, B., Peters, T., .. \& \& Gekeler, F. (2011). Transcorneal electrical stimulation for patients with retinitis pigmentosa: A prospective, randomized, sham-controlled exploratory study. Investigative Ophthalmology \& Visual Science, 52(7), 44854496.

Schmidt, S., Mante, A., Rönnefarth, M., Fleischmann, R., Gall, C., \& Brandt, S. A. (2013). Progressive enhancement of alpha activity and visual function in patients with optic neuropathy: A two-week repeated session alternating current stimulation study. Brain Stimulation, 6(1), 87-93.

Sehic, A., Guo, S., Cho, K. S., Corraya, R. M., Chen, D. F., \& Utheim, T. P. (2016). Electrical Stimulation as a Means for Improving Vision. American Journal of Pathology, 186 (11), 2783-2797.

Shinoda, K., Imamura, Y., Matsuda, S., Seki, M., Uchida, A., Grossman, T., \& Tsubota, K. (2008). Transcutaneous Electrical Retinal Stimulation Therapy for Age-Related Macular Degeneration. The Open Ophthalmology Journal, 2, 132-136.

Spiegel, D. P., Byblow, W. D., Hess, R. F., \& Thompson, B. (2013). Anodal transcranial direct current stimulation transiently improves contrast sensitivity and normalizes visual cortex activation in individuals with amblyopia. Neurorehabilitation and Neural Repair, 27(8), 760-769.

Spiegel, D. P., Li, J., Hess, R. F., Byblow, W. D., Deng, D., Yu, M., \& Thompson, B. (2013). Transcranial direct current stimulation enhances recovery of stereopsis in adults with amblyopia. Neurotherapeutics, 10(4), 831-839. 


\section{Appendix 1}

Appendix 1 Electrical Stimulation in visual conditions

\begin{tabular}{|c|c|c|c|c|c|c|c|c|}
\hline Year & $\begin{array}{l}\text { First } \\
\text { Author }\end{array}$ & Pathology & $\begin{array}{l}\text { Type } \\
\text { of } \\
\text { Stimu- } \\
\text { lation }\end{array}$ & $\begin{array}{l}\text { Time } \\
\text { of } \\
\text { Stimu- } \\
\text { lation }\end{array}$ & $\begin{array}{l}\text { General } \\
\text { Outcome }\end{array}$ & $\begin{array}{l}\text { Number } \\
\text { of } \\
\text { Patients } \\
\text { (patients } \\
\text { receiving } \\
\text { active } \\
\text { treatment) }\end{array}$ & $\begin{array}{l}\text { Side Effects } \\
\text { reported }\end{array}$ & $\begin{array}{l}\text { Level } \\
\text { of } \\
\text { Evidence }\end{array}$ \\
\hline 2010 & Gall & $\begin{array}{l}\text { Optic nerve } \\
\text { damage }\end{array}$ & rtACS & $\begin{array}{l}30-40 \mathrm{~min} \\
\times 10\end{array}$ & $\begin{array}{l}\text { VF, VA, VEP, } \\
\text { VOSP }\end{array}$ & $1(1)$ & not reported & IV \\
\hline 2011 & Fedorov & $\begin{array}{l}\text { Optic nerve } \\
\text { damage }\end{array}$ & rtACS & $\begin{array}{l}25-40 \mathrm{~min} \\
\times 10\end{array}$ & VF VA & $446(446)$ & $\begin{array}{l}10 \% \text { minor pain, local } \\
\text { warmth, vision blurring } \\
\text { effects for } 1-2 \text { min; }<5 \% \text { weak } \\
\text { headache, drowsiness, poor } \\
\text { sleep. Occasionally noticed } \\
\text { blood pressure fluctuation } \\
\text { and general fatigue. }\end{array}$ & IV \\
\hline 2011 & Gall & $\begin{array}{l}\text { Optic } \\
\text { neuropathy }\end{array}$ & rtACS & $\begin{array}{l}20-40 \mathrm{~min} \\
\times 10\end{array}$ & VF, NEI VFQ & $42(24)$ & not reported & II \\
\hline 2011 & Sabel & $\begin{array}{l}\text { Optic } \\
\text { neuropathy }\end{array}$ & rtACS & $\begin{array}{l}40 \min \times \\
10\end{array}$ & VF & $22(12)$ & $\begin{array}{l}5 \text { cutaneous sensations, } 4 \\
\text { sleeping difficulties, } 1 \\
\text { spontaneous phosphenes } \\
\text { independent of stimulation } \\
\text { during the } 10 \text {-day treatment } \\
\text { course }\end{array}$ & II \\
\hline 2013 & Schmidt & $\begin{array}{l}\text { Optic } \\
\text { neuropathy }\end{array}$ & rtACS & $\begin{array}{l}25-40 \mathrm{~min} \\
\times 10\end{array}$ & EEG, VF & $24(18)$ & 3 light frontal headache & III \\
\hline 2014 & Bola & $\begin{array}{l}\text { Optic nerve } \\
\text { damage }\end{array}$ & rtACS & $\begin{array}{l}40 \min \times \\
10\end{array}$ & VA, VF, EEG & $15(7)$ & not reported & III \\
\hline 2016 & Gall & $\begin{array}{l}\text { Optic nerve } \\
\text { damage }\end{array}$ & rtACS & $\begin{array}{l}25-50 \min \\
\times 10\end{array}$ & VF, EEG & $82(45)$ & $\begin{array}{l}2 \text { transient vertigo, } 1 \\
\text { persistent vertigo for } 0,5 \mathrm{hrs} \text {, } \\
1 \text { temporary dizziness, } 8 \text { mild } \\
\text { headaches, } 20 \text { cutaneous } \\
\text { sensations, } 1 \text { back pain and } \\
\text { stiff neck (drop-out) }\end{array}$ & I \\
\hline 2008 & Shinoda & $\begin{array}{l}\text { wet and dry } \\
\text { AMD }\end{array}$ & tpES & $\begin{array}{l}20 \min \times \\
20\end{array}$ & VA, VF & $21(21)$ & $\begin{array}{l}1 \text { "serious" contact dermatitis } \\
\text { on both superior lids }\end{array}$ & IV \\
\hline 2013 & $\begin{array}{l}\text { Anas- } \\
\text { tassiou }\end{array}$ & dry AMD & tpES & $\begin{array}{l}640 \sec \times \\
5\end{array}$ & $\begin{array}{l}\text { VA, CS and } \\
\text { macular } \\
\text { sensitivity }\end{array}$ & $22(12)$ & none & II \\
\hline 2015 & Chaikin & $\begin{array}{l}\text { wet and dry } \\
\text { AMD }\end{array}$ & tpES & $\begin{array}{l}35 \min x \\
2-10\end{array}$ & VA & $17(17)$ & not reported & IV \\
\hline 2018 & $\begin{array}{l}\text { Gil- } \\
\text { Carrasco }\end{array}$ & Glaucoma & tpES & $\begin{array}{l}40 \min \times \\
10\end{array}$ & IOP & 46 (58 eyes) & not reported & III \\
\hline 2006 & Fujikado & NAION, TON & tcES & $30 \min \times 1$ & $\mathrm{VA}, \mathrm{VF}$ & $8(8)$ & $\begin{array}{l}8 \text { mild superficial punctate } \\
\text { keratophaty }\end{array}$ & IV \\
\hline 2007 & Inomata & $\begin{array}{l}\text { Retinal Artery } \\
\text { Occlusion }\end{array}$ & tcES & $30 \min \times 3$ & VA, VF, ERG & $3(3)$ & 3 superficial keratitis & IV \\
\hline 2011 & Oono & $\begin{array}{l}\text { Retinal Artery } \\
\text { Occlusion }\end{array}$ & tcES & $30 \min \times 1$ & Brain activity & $5(5)$ & not reported & IV \\
\hline 2011 & Schatz & $\begin{array}{l}\text { Retinitis } \\
\text { Pigmentosa }\end{array}$ & tcES & $30 \min \times 6$ & $\begin{array}{l}\text { ERG, VF, } \\
\text { OCT, VA, DA, } \\
\text { color } \\
\text { discrimination }\end{array}$ & $24(8)$ & 2 foreign body sensasition & III \\
\hline 2013 & Naycheva & $\begin{array}{l}\text { Retinal Artery } \\
\text { Occlusion }\end{array}$ & tcES & $30 \min \times 6$ & VA, VF, ERG & $13(10)$ & 3 foreign body sensatons & III \\
\hline 2013 & Ozeki & $\begin{array}{l}\text { Best } \\
\text { Vitelliform } \\
\text { Macular } \\
\text { Dystrophy }\end{array}$ & tcES & $30 \min \times 2$ & VA, VF & $1(1)$ & 1 transient superficial keratitis & IV \\
\hline
\end{tabular}


Appendix 1 (Continued)

\begin{tabular}{|c|c|c|c|c|c|c|c|c|}
\hline Year & $\begin{array}{l}\text { First } \\
\text { Author }\end{array}$ & Pathology & $\begin{array}{l}\text { Type } \\
\text { of } \\
\text { Stimu- } \\
\text { lation }\end{array}$ & $\begin{array}{l}\text { Time } \\
\text { of } \\
\text { Stimu- } \\
\text { lation }\end{array}$ & $\begin{array}{l}\text { General } \\
\text { Outcome }\end{array}$ & $\begin{array}{l}\text { Number } \\
\text { of } \\
\text { Patients } \\
\text { (patients } \\
\text { receiving } \\
\text { active } \\
\text { treatment) }\end{array}$ & $\begin{array}{l}\text { Side Effects } \\
\text { reported }\end{array}$ & $\begin{array}{l}\text { Level } \\
\text { of } \\
\text { Evidence }\end{array}$ \\
\hline 2013 & $\begin{array}{l}\text { Robles- } \\
\text { Camarillo }\end{array}$ & $\begin{array}{l}\text { Retinitis } \\
\text { Pigmentosa, } \\
\text { Usher } \\
\text { Syndrome } \\
\text { type I or II }\end{array}$ & tcES & $\begin{array}{l}45 \min \times \\
52\end{array}$ & VA, VF & $28(28)$ & none & IV \\
\hline 2017 & Schatz & $\begin{array}{l}\text { Retinitis } \\
\text { Pigmentosa }\end{array}$ & tcES & $\begin{array}{l}30 \min \times \\
52\end{array}$ & $\begin{array}{l}\text { VA, VF, ERG, } \\
\text { DA, IOP }\end{array}$ & $52(32)$ & 31 dry eyes symptoms & II \\
\hline 2018 & Bittner & $\begin{array}{l}\text { Retinitis } \\
\text { Pigmentosa }\end{array}$ & tcES & $30 \min \times 6$ & $\begin{array}{l}\text { Retrobulbar } \\
\text { ocular haemo- } \\
\text { dynamics in } \\
\text { the CRA, } \\
\text { RBF, VA, CS, } \\
\text { VF, ERG, } \\
\text { OCT }\end{array}$ & $21(7)$ & $\begin{array}{l}3 \text { small brief electrical shock } \\
\text { when there was a loss of } \\
\text { connection with the ground } \\
\text { electrode, } 1 \text { when DTL } \\
\text { electrode lost contact with the } \\
\text { cornea and was reapplied } \\
\text { (discomfort at the moment of } \\
\text { the shock). }\end{array}$ & III \\
\hline 2018 & Bittner & $\begin{array}{l}\text { Retinitis } \\
\text { Pigmentosa }\end{array}$ & tcES & $30 \min \times 6$ & $\mathrm{VA}, \mathrm{CS}, \mathrm{VF}$ & $3(3)$ & not reported & IV \\
\hline 2018 & Ota & $\begin{array}{l}\text { Open-angle } \\
\text { Glaucoma }\end{array}$ & tcES & $\begin{array}{l}30 \min \times \\
6-27\end{array}$ & VF & $4(4)$ & not reported & IV \\
\hline 2014 & Camilleri & Myopia & $\begin{array}{l}\text { hf- } \\
\text { tRNS }\end{array}$ & $25 \min \times 8$ & $\mathrm{VA}, \mathrm{CS}$ & $16(8)$ & not reported & III \\
\hline 2014 & Campana & Amblyopia & $\begin{array}{l}\text { hf- } \\
\text { tRNS }\end{array}$ & $25 \min \times 8$ & VA, CS & $7(7)$ & not reported & IV \\
\hline 2016 & Camilleri & Myopia & $\begin{array}{l}\text { hf- } \\
\text { tRNS }\end{array}$ & $25 \min \times 8$ & $\mathrm{VA}, \mathrm{CS}$ & $30(10)$ & not reported & III \\
\hline 2018 & Moret & Amblyopia & $\begin{array}{l}\text { hf- } \\
\text { tRNS }\end{array}$ & $25 \min \times 8$ & $\mathrm{VA}, \mathrm{CS}$ & $20(10)$ & not reported & III \\
\hline 2011 & Halko & Hemianopia & $\mathrm{tDCS}$ & $\begin{array}{l}30 \min \times \\
72\end{array}$ & fMRI & $1(1)$ & not reported & IV \\
\hline 2011 & Plow & Hemianopia & $\mathrm{tDCS}$ & $\begin{array}{l}30 \min \times \\
72\end{array}$ & $\mathrm{VF}$ & $2(1)$ & none & IV \\
\hline 2012 & Plow & VFLoss & $\mathrm{tDCS}$ & $\begin{array}{l}30 \min \times \\
72\end{array}$ & VF & $8(4)$ & none & III \\
\hline 2012 & Plow & Hemianopia & $\mathrm{tDCS}$ & $\begin{array}{l}30 \min \times \\
72\end{array}$ & VF & $12(4)$ & none & III \\
\hline 2013 & Cowey & Hemianopia & $\mathrm{tDCS}$ & $15 \min \times 3$ & Blindsight & $1(1)$ & not reported & IV \\
\hline 2013 & Olma & $\begin{array}{l}\text { Homonimous } \\
\text { VF Defects }\end{array}$ & $\mathrm{tDCS}$ & $\begin{array}{l}20 \min \times \\
10\end{array}$ & $\begin{array}{l}\text { Motion } \\
\text { perception }\end{array}$ & $12(12)$ & 1 skin irritation, 1 chest pain & III \\
\hline 2013 & Spiegel & Amblyopia & $\mathrm{tDCS}$ & $15 \min \times 2$ & $\mathrm{CS}$ & $13(13)$ & $\begin{array}{l}1 \text { patient withdraw for } \\
\text { discomfort }\end{array}$ & IV \\
\hline 2013 & Spiegel & Amblyopia & $\mathrm{tDCS}$ & $\begin{array}{l}15 \min \times \\
10\end{array}$ & Stereopsis & $16(16)$ & $\begin{array}{l}\text { slight tingling sensation } \\
\text { under the electrodes }\end{array}$ & III \\
\hline 2016 & Ding & Amblyopia & $\mathrm{tDCS}$ & $20 \min \times 3$ & VEP, CS & $48(21)$ & not reported & III \\
\hline 2016 & Kim & $\begin{array}{l}\text { Post-stroke } \\
\text { Visual } \\
\text { Perception } \\
\text { Degradation }\end{array}$ & tDCS & $\begin{array}{l}20 \min \times \\
30\end{array}$ & MVPT, FIM & $30(15)$ & not reported & III \\
\hline 2017 & Alber & Hemianopia & $\mathrm{tDCS}$ & $\begin{array}{l}20 \min \times \\
10\end{array}$ & $\mathrm{VF}$ & $14(7)$ & $\begin{array}{l}\text { Occasional itching or tingling } \\
\text { underneath the electrodes }\end{array}$ & III \\
\hline 2017 & Matteo & Hemianopia & $\mathrm{tDCS}$ & $\begin{array}{l}30 \min \times \\
40\end{array}$ & VF & $2(2)$ & none & IV \\
\hline
\end{tabular}

Notes: $\mathrm{VF}=$ Visual Field, VA = Visual Acuity, VEP = Visual Evoked Potentials, VOSP = Visual Object and Space Perception battery, NEI VFQ= National Eye Institute Visual Functioning Questionnaire, CS =Contrast Sensitivity, IOP=Intra-Ocular Pressure, ERG $=$ Electroretinography, OCT $=$ Optical Coherence Tomography, DA = Dark Adaptation, $\mathrm{CRA}=\mathrm{Central}$ Retinal Artery, RBF $=$ Retinal Blood Flow, $\mathrm{fMRI}=$ functional Magnetic Resonance Imaging, MVPT = Motor-free Visual Perception Test, FIM = Functional Independence Measure scale. 